\title{
Beurteilung der Versorgungslage von Milchkühen in der frühen Laktation anhand der Milchinhaltsstoffe
}

\section{Assessment of the protein and energy supply of early lactating dairy cows based on milk components}

\author{
Karin Ornetsmüller ${ }^{1}$, Leonhard Gruber ${ }^{1,2}$, Marco Horn ${ }^{3}$, Werner Zollitsch ${ }^{1 *}$ \\ ${ }^{1}$ Universität für Bodenkultur Wien, Department für Nachhaltige Agrarsysteme, Institut für Nutztierwissenschaften, Gregor-Mendel-Straße 33, \\ 1180 Wien, Österreich \\ ${ }^{2}$ Höhere Bundeslehr- und Forschungsanstalt für Landwirtschaft Raumberg-Gumpenstein, Institut für Nutztierforschung, Altirdning 11, 8952 \\ Irdning-Donnersbachtal, Österreich \\ ${ }^{3}$ Landwirtschaftskammer Niederösterreich, Wiener Straße 64, 3100 St. Pölten, Österreich
}

* Korrespondierender Autor: werner.zollitsch@boku.ac.at

Received: 24 April 2019, received in revised form: 20 August 2019, accepted: 23 August 2019

\section{Zusammenfassung}

Da sich die Einschätzung der Versorgungslage von Milchkühen in der Praxis oft schwierig gestaltet, wird dafür seit einigen Jahren eine grafische Darstellung auf Basis des Milcheiweiß- und Harnstoffgehaltes als Management-Tool verwendet. Dabei stellt sich die Frage, wie gut die Beurteilungen dieser sogenannten 9-Felder-Tafel und ihrer aktualisierten Form, der 6-Felder-Tafel, mit der tatsächlichen Protein- und Energieversorgungslage übereinstimmen. Um dies zu ermitteln, wurden Daten aus einem Fütterungsversuch in die FelderTafeln eingeordnet und mithilfe eines Chi-Quadrat-Anpassungstests, der Berechnung von Sensitivität, Spezifität sowie der LikelihoodRatios auf die Zuverlässigkeit der Einschätzung hin überprüft. Eine Optimalversorgung wurde für den Bereich einer Aufnahme von $\pm 10 \%$ des Tagesbedarfs angenommen. Dabei konnte innerhalb der ersten zehn Laktationswochen die 6-Felder-Tafel mit 27,6 \% korrekten Einschätzungen etwas bessere Ergebnisse erzielen als die 9-Felder-Tafel mit 17,2 \%. Die Sensitivität reichte bei der 9-Felder-Tafel von 0,00 bis 0,50, bei der 6-Felder-Tafel von 0,00 bis 0,56. Ergänzend wurden die falschen Beurteilungen mit ihrer tatsächlichen Einordnung verglichen. Dabei konnte festgestellt werden, dass sich diese häufig nicht in den angrenzenden Feldern befinden, weshalb eine Adaptierung der Grenzwerte zu keiner wesentlichen Verbesserung der Zuverlässigkeit führt. Aufgrund gravierender Fehleinschätzungen können beide Felder-Tafeln nicht für eine Verwendung zur Einzeltierbeurteilung in der Praxis empfohlen werden.

Schlagworte: Felder-Tafel, Milcheiweiß, Milchharnstoff, Milchfett, Fett/Eiweiß-Quotient

\section{Summary}

For the on-farm assessment of protein and energy supply of dairy cows, specific management tools based on milk protein and urea content are used. In order to determine the degree of agreement between estimates from the so-called 9-fields table or the updated 6-fields table and the actual protein and energy supply, data from a feeding trial were used (chi-square fit test, sensitivity, specificity and likelihood ratios). The optimum supply range for both crude protein and energy was defined within $\pm 10 \%$ of daily requirement. For the first ten weeks of lactation the proportions of correct estimates were $17.2 \%$ and $27.6 \%$ for the 9-fields and the 6-fields table, respectively. Sensitivity ranged from 0.00 to 0.50 and from 0.00 to 0.56 , respectively. In addition, the false assessments were compared with their effective classification. It was found that these are not always located in the adjacent fields. Therefore a mere adaptation of thresholds for milk urea, milk protein or the fat to protein ratio will not substantially improve the reliability of the assessment. Due to the high proportions of seriously false estimates, the tools tested in the present study cannot be recommended for assessment of energy and protein supply of individual dairy cows.

Keywords: milk protein, milk urea, milk fat, fat to protein ratio, management tool 


\section{Einleitung}

Der bedarfsdeckenden Versorgung von Milchkühen kommt aus mehreren Gründen große Bedeutung zu: Nährstoffe, die ungenutzt wieder ausgeschieden werden, sollen sowohl aus ökologischen Gründen (Belastung von Tier und Umwelt) (Gerber et al., 2014; Makkar, 2016) als auch aus ökonomischen Gründen (Kosten v. a. eiweißreicher Futtermittel) (Losand et al., 2016) auf ein Minimum reduziert werden. Vor allem zu Beginn der Laktation sind fütterungsbedingte Stoffwechselstörungen zu erwarten (Seggewiß, 2004), weshalb einer ausgeglichenen Fütterung große Bedeutung zukommt. Auch steigt die Achtsamkeit der Konsumenten für Produkte, die nachhaltig und tiergerecht produziert werden (Tamminga, 2003).

In der Praxis gestaltet sich eine optimale Versorgung mit Protein und Energie schwierig, da die exakte Futteraufnahme nur schwer festgestellt werden kann (Hof et al., 1997; Eicher et al., 1999; Friggens et al., 2007). Ein einfaches und günstiges Management-Tool, das regelmäßig Informationen zur Versorgungslage von Milchkühen bietet, wäre deshalb von großem Wert für die landwirtschaftliche Praxis (Hof et al., 1997). Die Nutzung der Milchinhaltsstoffe wäre, wenn die Einschätzungsgenauigkeit hoch genug ist, eine simple, verlässliche und preiswerte Methode (Friggens et al., 2007). Eine restriktive Energieversorgung führt bei Milchkühen zu sinkenden Protein- und steigenden Harnstoffgehalten in der Milch, eine übermäßige Energieaufnahme kehrt diese Effekte um (Kirchgeßner et al., 1986). Ist wenig
Protein bzw. Stickstoff im Pansen verfügbar, wird die Ausscheidung durch Recycling des Stickstoffs minimiert und der Harnstoffgehalt in der Milch sinkt (Hof et al., 1997). Durch diese Wiederverwertung wird die Proteinversorgung bestmöglich aufrechterhalten und der Eiweißgehalt der Milch bleibt annähernd gleich. Tritt Protein im Überschuss auf, steigt die Ausscheidung als Harnstoff an (Spohr und Wiesner, 1991). Ist ausreichend Energie vorhanden, bleibt der Proteingehalt der Milch konstant. Restriktionen der Versorgung führen dabei zu deutlicheren Ausprägungen als Überschüsse (Kirchgeßner et al., 1986).

Eine grafische Darstellung zur Einschätzung der Versorgung anhand des Eiweiß- und Harnstoffgehaltes der Milch, entwickelt von Spohr und Wiesner (1991), wird seit circa 20 Jahren in Form einer Auswertung der Ergebnisse der Milchleistungskontrolle auf den österreichischen Höfen angeboten. Die sogenannte 9-Felder-Tafel soll die Landwirte bei der Kontrolle des Fütterungsmanagements unterstützen und auf eine Fehlversorgung hinweisen. $\mathrm{Zu}$ nehmend gibt es aber auch Zweifel, wie gut diese Einteilung für die aktuellen Hochleistungstiere zutrifft, da deren Leistung durch Zuchtfortschritt und optimiertes Management steigt und dabei der Eiweißgehalt der Milch sinkt. Ein neues Bewertungsschema, die 6-Felder-Tafel, soll eine, dem Potenzial der Kühe besser entsprechende, grafische Einschätzungsform bieten (Losand et al., 2016). Die 9-Felder-Tafel geht dabei von einem Optimalversorgungsbereich bei 3,2 \% bis 3,8\% Milcheiweiß und 150-300 mg/l Harnstoff aus (Abbildung 1 nach Spohr und Wiesner

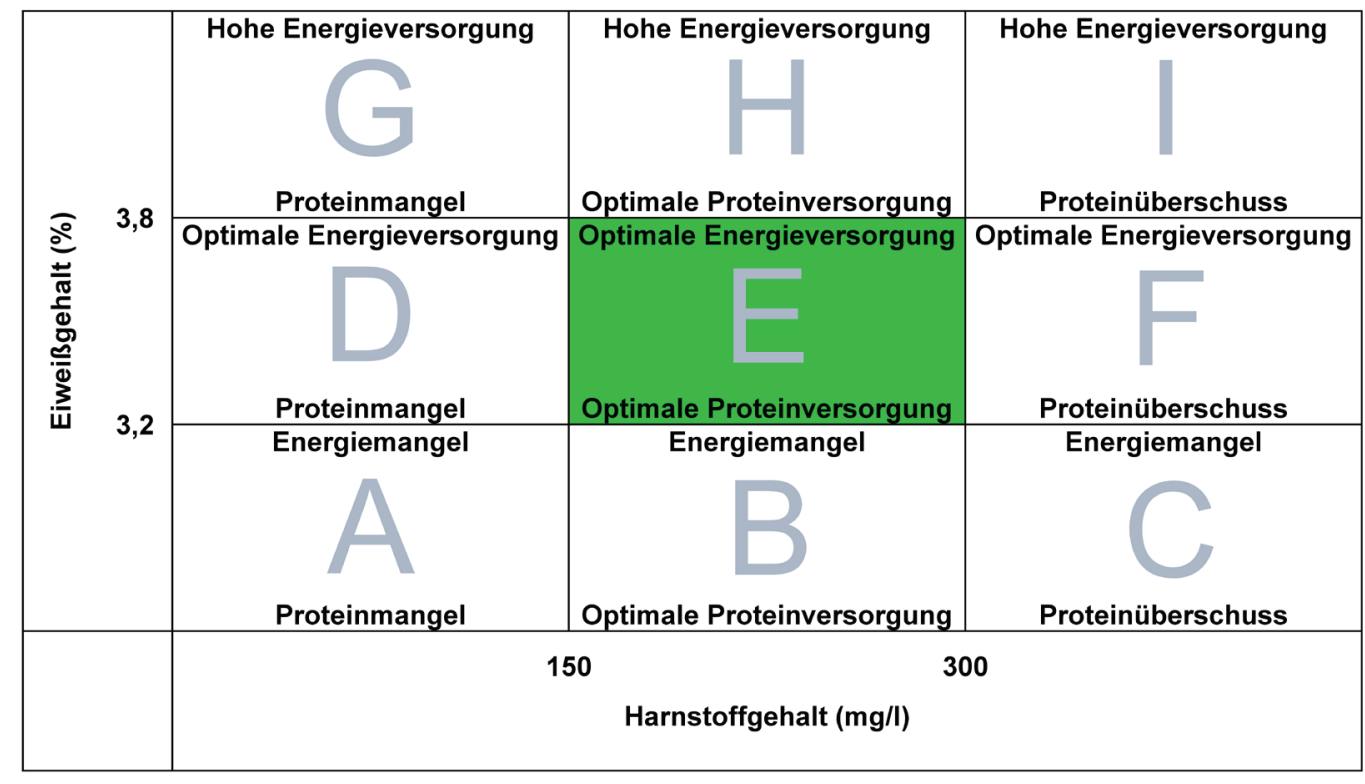

Abbildung 1. 9-Felder-Tafel nach Spohr und Wiesner (1991) und Seggewiß (2004)

Figure 1. 9-field table according to Spohr and Wiesner (1991) and Seggewiß (2004) 
(1991)). Die 6-Felder-Tafel beurteilt die Energieversorgung nach dem Fett/Eiweiß-Quotient (FEQ) mit einer ausreichenden Versorgung bei einem Wert $\leq 1,4$. Wegen seiner höheren Stabilität gegenüber Einflüssen der Milchmenge scheint er ein besserer Indikator für die Energieversorgung zu sein (Losand et al., 2016). Die unteren und oberen Harnstoffgrenzen wurden bei 150 und $250 \mathrm{mg} / \mathrm{l}$ Harnstoff fixiert (Abbildung 2 nach Losand et al., 2016).

Belastbare Daten zur Übereinstimmung der Beurteilung gemäß diesen Tafeln mit der tatsächlichen, unter kontrollierten Bedingungen erhobenen Versorgungssituation liegen bisher nicht vor. Deshalb sollte im Rahmen der vorliegenden Arbeit durch eine Einordnung der Daten aus einem Fütterungsversuch in die Felder-Tafeln den Fragen nachgegangen werden, wie gut die Einschätzung anhand der Milchinhaltsstoffe mit jener nach der tatsächlichen Versorgungslage übereinstimmt, in welchen Feldern der Tafeln die korrekte Beurteilung am höchsten ist und in welchen sich die nicht korrekt eingeschätzten Beobachtungen befinden.

\section{Material und Methoden}

Die Daten entstammen einem Fütterungsversuch, der von 2005 bis 2009 an der Bundeslehr- und Forschungsanstalt Raumberg-Gumpenstein nach einem zwei-faktoriellen Design mit drei Energie- und drei Proteinstufen $(3 \times 3)$ durchgeführt wurde (Gruber et al., 2019; unveröffentlichte Ergebnisse).

Der Versuch wurde mit 57 Milchkühen durchgeführt, die sich zumindest in der zweiten Laktation befanden. In Tabelle 1 ist die Aufteilung der Tiere in die verschiedenen Gruppen (1, 2, 3 in Kombination mit N, M, H) des Ver- suches ersichtlich $(1 / \mathrm{N}=$ niedrige, $2 / \mathrm{M}=$ mittlere, $3 / \mathrm{H}$ = hohe Versorgung). In den Energiegruppen 1, 2 und 3 befanden sich jeweils 19 Tiere, in den Proteingruppen N, $\mathrm{M}$ und $\mathrm{H}$ befanden sich 14, 18 und 25 Tiere. Es wurden milchbetonte Kühe der Rassen Holstein $(\mathrm{n}=27)$ und Brown Swiss ( $\mathrm{n}=14)$ ausgewählt sowie Kreuzungen dieser zwei Rassen $(\mathrm{n}=12)$ und Kreuzungen mit der Rasse Fleckvieh $(\mathrm{n}=4)$.

Den Versuchstieren wurde das Grundfutter $(50 \%$ Grassilage, $30 \%$ Maissilage, $20 \% \mathrm{Heu}$ ) dreimal täglich zur freien Aufnahme vorgelegt. Die Grundfutteraufnahme wurde täglich erhoben, zweimal pro Tag erfolgte die Rückwaage der Futterreste. Die Kraftfuttermittel wurden über eine Transponderstation gefüttert. Die Menge des Kraftfutters wurde dreimal wöchentlich der aufgenommenen Trockenmasse des Grundfutters angepasst. Die Energie-Stufen wurden über unterschiedliche Konzentratfutter-Anteile der Ration variiert, und zwar 20, 35, $50 \%$ der TM in den Gruppen 1, 2 und 3, welche durchschnittlich 105, 133 und $147 \mathrm{MJ} \mathrm{NEL/d}$ aufnahmen. Die unterschiedlichen Protein-Stufen wurden durch verschiedene Anteile eines energie- bzw. proteinbetonten Konzentratfutters (EKF, PKF) erzielt. Das EKF wies einen Anteil von $35 \%$ Mais, $33 \%$ Winterweizen, $30 \%$ Wintergerste und $2 \%$ Rapsöl auf. Das PKF setzte sich aus $48 \%$ Ackerbohne, $30 \%$ Rapsextraktionsschrot, 20 \% Maiskleber und $2 \%$ Rapsöl zusammen. Die Anteile an EKF bzw. PKF betrugen im Durchschnitt $100 \%$ bzw. $0 \%, 77 \%$ bzw. $33 \%$ und $53 \%$ bzw. $47 \%$ in den Gruppen N, M und H. Damit wurde ein Proteingehalt der Gesamtration von durchschnittlich 115, 136 und 157 g XP/kg TM erreicht. Der Nährstoffgehalt der Rationen aus dem Fütterungsversuch und die realisierte Futter-, Energie- und Proteinaufnahme sind in Tabelle 2 angegeben.

Tabelle 1. Anzahl der Tiere im Versuch $(1 / \mathrm{N}=$ niedrige, $2 / \mathrm{M}=$ mittlere, $3 / \mathrm{H}=$ hohe Versorgung mit Energie/Protein)

Table 1 . Number of animals in the trial $(1 / \mathrm{N}=$ low, $2 / \mathrm{M}=$ medium, $3 / \mathrm{H}=$ high supply with energy/protein)

\begin{tabular}{|c|c|c|c|c|}
\hline Energie & & Protein & & Gesamt \\
\hline & $\mathrm{N}$ & $\mathrm{M}$ & $\mathrm{H}$ & \\
\hline 1 & $\begin{array}{c}4 \\
(2 \mathrm{HO}, 2 \mathrm{BS})\end{array}$ & $\begin{array}{c}5 \\
(3 \mathrm{HO}, 2 \mathrm{BS} \times \mathrm{HO})\end{array}$ & $\begin{array}{c}10 \\
(7 \mathrm{HO}, 2 \mathrm{BS}, 1 \mathrm{BS} \times \mathrm{HO})\end{array}$ & 19 \\
\hline 2 & $\begin{array}{c}5 \\
(3 \mathrm{HO}, 2 \mathrm{BS})\end{array}$ & $\stackrel{7}{(1 \mathrm{HO}, 1 \mathrm{BS}, 4 \mathrm{BS} \times \mathrm{HO}, 1 \mathrm{HO} \times \mathrm{FV})}$ & $\begin{array}{c}7 \\
(3 \mathrm{HO}, 3 \mathrm{BS}, 1 \mathrm{BS} \times \mathrm{HO})\end{array}$ & 19 \\
\hline 3 & $\begin{array}{c}5 \\
(4 \mathrm{HO}, 1 \mathrm{BS} \times \mathrm{HO})\end{array}$ & $\begin{array}{c}6 \\
(2 \mathrm{HO}, 1 \mathrm{BS}, 1 \mathrm{BS} \times \mathrm{HO}, 1 \mathrm{HO} \times \mathrm{FV}, 1 \\
\mathrm{BS} \times \mathrm{FV})\end{array}$ & $\begin{array}{c}8 \\
(2 \mathrm{HO}, 3 \mathrm{BS}, 3 \mathrm{BS} \times \mathrm{HO})\end{array}$ & 19 \\
\hline Gesamt & 14 & 18 & 25 & 57 \\
\hline
\end{tabular}

$\mathrm{HO}=$ Holstein; BS = Brown Swiss; FV = Fleckvieh 
Die energiekorrigierte Milchleistung lag für die neun Gruppen zwischen 22,9 und 32,3 kg/d (LS-Mittel; RMSE = 4,4). Der durchschnittliche Milcheiweißgehalt der Gruppen variierte von $2,88 \%$ bis $3,39 \%$ (RMSE $=0,25)$, der Fettgehalt von $4,19 \%$ bis $4,98 \%(\mathrm{RMSE}=0,72)$ und der Milchharnstoffgehalt von $126 \mathrm{mg} / \mathrm{l}$ bis $269 \mathrm{mg} / \mathrm{l}$ (RMSE $=52$ ).

Für die vorliegende Auswertung wurden die Daten aus dem ersten Laktationsdrittel der ersten Laktation des Versuchszeitraumes verwendet. Die Daten wurden für die Auswertung auf jene Messungen beschränkt, für die alle benötigten Informationen vorlagen. Dabei wurden von 41 Kühen durchschnittlich je 4,0 $( \pm 1,2)$ Messungen verwendet, die zwischen der 2. und 10. Laktationswoche durchgeführt wurden. Jede der insgesamt 163 Messungen wurde nach den Kriterien der Felder-Tafeln den zutreffenden Feldern zugeordnet. Von jedem Tier wurde bei jeder Messung jeweils eine Rohprotein- bzw. Energiebilanz auf Basis der Angaben von Kirchgeßner (1992), der GfE (2001) bzw. Urdl et al. (2015) erstellt. Die Versorgung mit am Duodenum verfügbarem Rohprotein (nXP) wird neben der Rohprotein (XP)-Aufnahme auch von dessen ruminaler Abbaubarkeit sowie der Energieversorgung der Symbionten im Pansen beeinflusst (Schwarz, 2014). Um den Effekt der Protein- von dem der Energieversorgung besser trennen zu können, wurde in der vorliegenden Arbeit daher nicht
nXP, sondern XP als Maßstab für die Proteinversorgung verwendet. Der optimale Versorgungsbereich sowohl für NEL als auch für XP wurde dabei als $\pm 10 \%$ des errechneten Tagesbedarfs angenommen. Darunter bzw. darüber liegende Werte gelten als unter- bzw. überversorgt.

Die Rohproteinbilanz wurde wie folgt berechnet: $\mathrm{XP}-$ Bilanz $=$ XP-Aufnahme $-\mathrm{XP}$-Bedarf

Für die Berechnung des Rohproteinbedarfs wurde auf die Angaben aus Kirchgeßner (1992) zurückgegriffen und der Bedarf aufgrund Lebendmasse, Milchmenge und Eiweißgehalt mit folgender Formel berechnet:

$$
\begin{gathered}
\text { XP-Bedarf } / \text { Tag }=(0,5 \times \mathrm{kg} \mathrm{LM}+150)+ \\
((15 \times \% \text { Eiweißgehalt Milch }+30) \times \mathrm{kg} \text { Milch })
\end{gathered}
$$

Die für Erhaltung, Milchbildung und Trächtigkeit benötigte Energie wurde nach Urdl et al. (2015) ermittelt. Die Energiebilanz wurde mithilfe folgender Formel erstellt:

Energiebilanz $=$ NEL-Aufnahme -

(NEL-Erhaltung + NEL-Milch + NEL-Trächtigkeit)

Der Energiebedarf wurde nach den Empfehlungen der Gesellschaft für Ernährungsphysiologie zur Energie- und Nährstoffversorgung der Milchkühe und Aufzuchtrinder kalkuliert (GfE, 2001).

Tabelle 2. Rationskriterien, Futter-, Energie- und Nährstoffaufnahme bei unterschiedlicher Energie- und Proteinversorgung (LS-Mittel) $(1 / \mathrm{N}=$ niedrige, $2 / \mathrm{M}=$ mittlere, $3 / \mathrm{H}=$ hohe Versorgung mit Energie/Protein)

Table 2. Dietary characteristics, feed, energy and nutrient intake for dietary treatments with different energy and protein intake (LS-means) (1/N

\begin{tabular}{|c|c|c|c|c|c|c|c|}
\hline \multirow[t]{2}{*}{ Parameter } & \multicolumn{3}{|c|}{ Energie-Versorgung } & \multicolumn{3}{|c|}{ Protein-Versorgung } & \multirow[t]{2}{*}{ RMSE } \\
\hline & 1 & 2 & 3 & $\mathrm{~N}$ & M & $\mathrm{H}$ & \\
\hline \multicolumn{8}{|c|}{ Kriterien der Gesamtration (in der T) } \\
\hline Kraftfutteranteil, \% & $16,1^{a}$ & $32,4^{\mathrm{b}}$ & $45,2^{c}$ & $30,3^{a}$ & $31,4^{\mathrm{ab}}$ & $32,0^{\mathrm{b}}$ & 3,7 \\
\hline $\mathrm{XP}, \mathrm{g} / \mathrm{kg}$ & $125^{\mathrm{a}}$ & $137^{\mathrm{b}}$ & $145^{\circ}$ & $115^{a}$ & $136^{\mathrm{b}}$ & $157^{\mathrm{c}}$ & 11 \\
\hline nXP, g/kg & $133^{\mathrm{a}}$ & $144^{\mathrm{b}}$ & $152^{\mathrm{c}}$ & $136^{a}$ & $143^{\mathrm{b}}$ & $150^{c}$ & 4 \\
\hline NEL, MJ & $6,06^{a}$ & $6,49^{\mathrm{b}}$ & $6,82^{\mathrm{c}}$ & 6,42 & 6,47 & 6,47 & 0,15 \\
\hline \multicolumn{8}{|c|}{ Futteraufnahme (pro Tag) } \\
\hline Grundfutter, $\mathrm{kg} \mathrm{T}$ & $14,53^{\mathrm{a}}$ & $13,84^{a}$ & $11,70^{\mathrm{b}}$ & 13,15 & 13,30 & 13,63 & 1,99 \\
\hline Kraftfutter, $\mathrm{kg} \mathrm{T}$ & $2,74^{a}$ & $6,65^{b}$ & $9,66^{c}$ & $6,08^{\mathrm{a}}$ & $6,30^{a}$ & $6,67^{b}$ & 0,87 \\
\hline Gesamtration, kg T & $17,25^{\mathrm{a}}$ & $20,48^{\mathrm{b}}$ & $21,34^{\mathrm{b}}$ & 19,21 & 19,58 & 20,29 & 2,34 \\
\hline Rohprotein, g & $2187^{\mathrm{a}}$ & $2841^{\mathrm{b}}$ & $3124^{c}$ & $2206^{a}$ & $2700^{\mathrm{b}}$ & $3246^{c}$ & 398 \\
\hline $\mathrm{nXP}, \mathrm{g}$ & $2307^{a}$ & $2964^{\mathrm{b}}$ & $3265^{c}$ & $2628^{a}$ & $2832^{\mathrm{b}}$ & $3077^{c}$ & 346 \\
\hline RNB, g & $-19,1$ & $-19,8$ & $-22,5$ & $-67,4^{a}$ & $-21,0^{\mathrm{b}}$ & $27,1^{c}$ & 26,9 \\
\hline NEL, MJ & $104,7^{\mathrm{a}}$ & $133,4^{\mathrm{b}}$ & $146,6^{c}$ & 124,7 & 127,6 & 132,3 & 15,1 \\
\hline
\end{tabular}
= low, $2 / \mathrm{M}=$ medium, $3 / \mathrm{H}$ = high supply with energy/protein)

$\mathrm{T}=$ Trockenmasse $\mathrm{XP}=$ Rohprotein; $\mathrm{nXP}=$ nutzbares Rohprotein am Duodenum; NEL = Netto-Energie-Laktation; RNB = ruminale N-Bilanz.

Unterschiedliche, hochgestellte Buchstaben kennzeichnen signifikant $(\mathrm{P}<0,05)$ voneinander verschiedene LS-Mittel. 


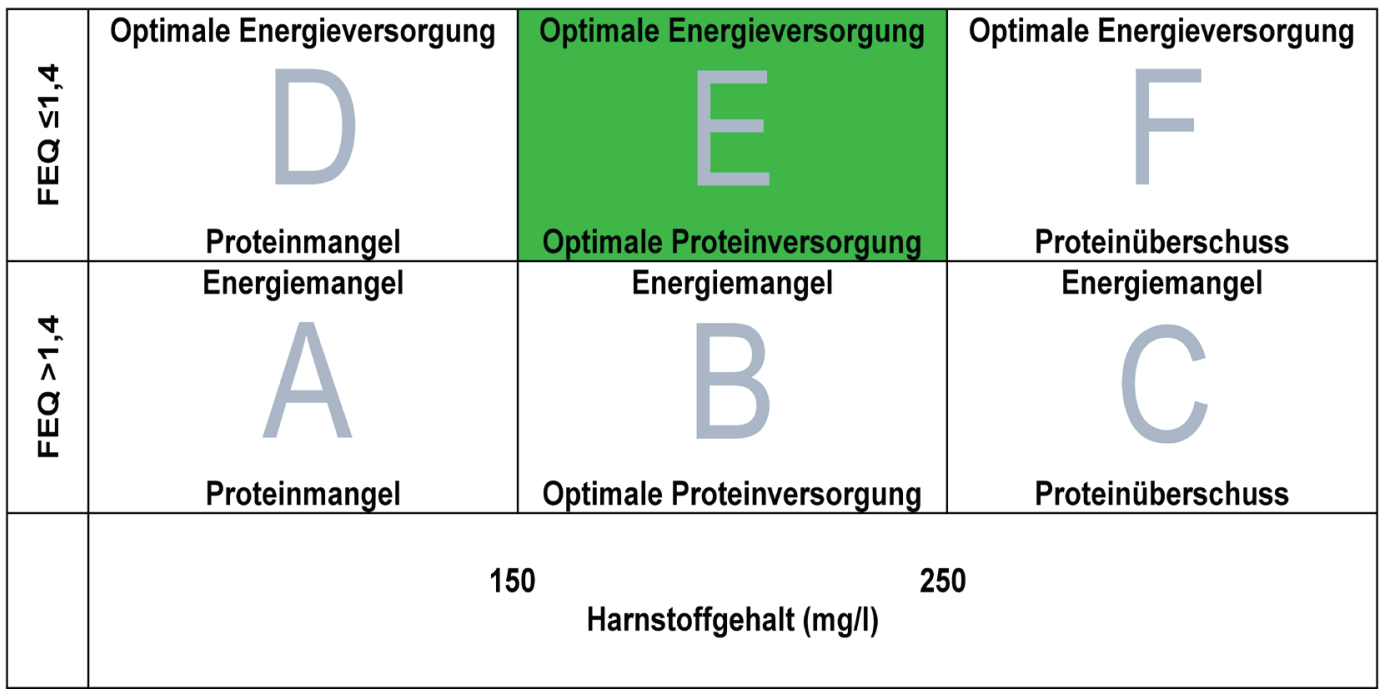

Abbildung 2. Vereinfachte Darstellung der 6-Felder-Tafel nach Losand et al. (2016). (Im Original werden die beiden Energiekategorien durch Verwendung des Eiweißgehaltes erneut geteilt, wodurch 12 Felder entstehen.)

Figure 2. Simplified representation of the 6-field table according to Losand et al. (2016). (In the original, the two energy categories are divided again by using the protein content, resulting in a total of 12 fields.)

Die mit der tatsächlichen Versorgungssituation korrespondierenden Gehalte an Milchinhaltsstoffen wurden in die Gruppen der 9- und 6-Felder-Tafel eingeteilt. Um festzustellen, ob die Verteilung der errechneten Versorgungsbilanz mit jenen der Felder-Tafeln kongruiert, wurden im Statistikprogramm SAS (Version 9.4, SAS Institute Inc., Cary, NC, USA) Chi-Quadrat-Anpassungstests durchgeführt.

Weiters wurden für alle Felder beider Tafeln jeweils Sensitivität und Spezifität ermittelt. Diese beiden Merkmale sind folgendermaßen definiert (Bender 2001; Firk et al., 2002):

$$
\begin{gathered}
\text { Sensitivität }=\mathrm{RP} /(\mathrm{RP}+\mathrm{FN}) \\
\text { Spezifität }=\mathrm{RN} /(\mathrm{RN}+\mathrm{FP})
\end{gathered}
$$

Richtig positiv $(\mathrm{RP})=$ richtig Feld $\mathrm{X}$ zugeordnet; Richtig negativ $(\mathrm{RN})=$ richtig nicht Feld $\mathrm{X}$ zugeordnet;

Falsch positiv $(\mathrm{FP})=$ falsch Feld $\mathrm{X}$ zugeordnet; Falsch negativ $(\mathrm{FN})=$ falsch nicht Feld X zugeordnet
Die „Likelihood-Ratio“ setzt Sensitivität und Spezifität in Relation zueinander und gibt an, wie sich die $\mathrm{Zu}$ ordnung in ein Tafel-Feld gemäß Milchinhaltsstoffen auf das Vorliegen einer tatsächlich vorhandenen Unter-, Optimal- oder Überversorgung (siehe oben) auswirkt. Die Kategorisierung nach Tabelle 4 ermöglicht eine näherungsweise Aussage über die Qualität des geprüften Tests. Berechnet werden die beiden Werte wie folgt:

Negative Likelihood-Ratio (LR-) = (1 - Sensitivität $) \div$ Spezifität Positive Likelihood-Ratio $\left(\mathrm{LR}_{+}\right)=$ Sensitivität $\div(1-$ Spezifität $)$

Dabei gibt LR- an, wie sich die Wahrscheinlichkeit der Zugehörigkeit einer Beobachtung in ein Tafel-Feld aufgrund der tatsächlichen Versorgungssituation bei $\mathrm{Zu}$ ordnung der jeweiligen Gemelksprobe in ein anderes Tafel-Feld verändert. LR+ drückt aus, wie sich die Wahr-

Tabelle 3. Aufteilung der Beobachtungen nach Energie- und Protein-Bilanz in den ersten zehn Laktationswochen in Prozent (\%) und deren Median (M) Table 3. Distribution of observations according to energy and protein balance in the first ten weeks of lactation in percent (\%) and their median (M)

\begin{tabular}{cccccc}
\hline Bedarfsdeckung & Energieversorgung & & \multicolumn{2}{c}{ Proteinversorgung } \\
\hline & $\%$ & $\mathrm{M}$ & $\%$ & $\mathrm{M}$ \\
$<90 \%$ & 31,3 & 81,4 & 33,1 & 78,7 \\
$90-110 \%$ & 46,0 & 101,9 & 42,3 & 99,8 \\
$>110 \%$ & 22,7 & 120,3 & 24,6 & 123,6 \\
\hline
\end{tabular}

Bedarfsdeckung: Gegenüberstellung von Aufnahme und Bedarf. Berechnung: Protein-Bedarf nach Kirchgessner (1992), Energie-Bedarf nach GfE (2001). 
Tabelle 4. Testaussage von positiver und negativer Likelihood-Ratio (LR+, LR-) nach Bender (2001)

Table 4. Test results of positive and negative likelihood ratio (LR+, LR-) according to Bender (2001)

\begin{tabular}{lcc}
\hline Testaussage & LR+ & LR- \\
\hline Sehr gut & $>10$ & $<0,1$ \\
Gut & $5-10$ & $0,1-0,2$ \\
Mäßig & $2-5$ & $0,2-0,5$ \\
Schlecht & $1-2$ & $0,5-1,0$ \\
\hline
\end{tabular}

scheinlichkeit der Zugehörigkeit einer Beobachtung in ein Tafel-Feld aufgrund der tatsächlichen Versorgungssituation bei Zuordnung der jeweiligen Gemelksprobe in dieses Tafel-Feld verändert (Bender, 2001).

\section{Ergebnisse und Diskussion}

Im Folgenden werden die Häufigkeiten einer korrekten Zuordnung von Gemelksproben zu den beiden Tafeln dargestellt und diskutiert. Danach erfolgt die Darstellung der Zuverlässigkeit der Einschätzungen gemäß den beiden Tafeln, bevor die fehlerhaften Zuordnungen näher betrachtet werden.

\subsection{Häufigkeit zutreffender Einschätzungen}

Die Einordnung der Beobachtungen nach dem Grad der Bedarfsdeckung ergab eine ähnliche Verteilung von Energie- als auch Proteinversorgung mit jeweils über $40 \%$ in der Gruppe $90-110 \%$ und unter $25 \%$ in der Gruppe $>110 \%$ (Tabelle 3).

Die 9-Felder-Tafel und die 6-Felder-Tafel wurden auf den Anteil der korrekten Einschätzungen durch Vergleich mit der tatsächlichen Protein- und Energieversorgung überprüft. Dabei konnte festgestellt werden, dass beide Tafeln bei Kombination beider Kriterien weniger als $30 \%$ der Beobachtungen richtig beurteilen. Der Anteil der korrekt

Tabelle 5. Richtige Einschätzungen der Felder-Tafeln in den ersten zehn Laktationswochen (LW)

Table 5. Proportion of correctly assigned estimates for the field tables in the first ten weeks of lactation (LW)

\begin{tabular}{lcc}
\hline & 9-Felder-Tafel & 6-Felder-Tafel \\
\hline Energie korrekt (\%) & 33,7 & 54,0 \\
Protein korrekt (\%) & 39,3 & 41,1 \\
Kombination korrekt (\%) & 17,2 & 27,6 \\
\hline
\end{tabular}

eingeschätzten Beobachtungen nach der 9-Felder-Tafel in dieser Arbeit beträgt 17,2 \% (Tabelle 5). Aufgrund des Milcheiweißgehaltes konnten 33,7 \% der Beobachtungen hinsichtlich Energieversorgung korrekt eingeschätzt werden, der Milchharnstoffgehalt taugte bei 39,3\% zur Beurteilung der Proteinversorgung. Der Chi-Quadrat-Anpassungstest bestätigte einen signifikanten Unterschied $(P<0,001)$ zwischen der erwarteten Verteilung auf Basis der tatsächlichen Versorgungslage und der nach den Milchinhaltsstoffen geschätzten Versorgungslage (Ergebnisse nicht dargestellt). Da sich in den Feldern H, I und F keine Einschätzungen nach den Milchinhaltsstoffen befanden (siehe Abbildung 3), wurden diese vor der Auswertung zusammengefasst. Dabei wurde Feld $\mathrm{H}$ zusammen mit Feld E und die Felder I und F wurden zusammen mit Feld C ausgewertet.

Der geringe Anteil korrekter Einschätzungen anhand der 9-Felder-Tafel in der vorliegenden Arbeit deckt sich mit den Ergebnissen von Seggewiß (2004), die in ihrer Arbeit einen Anteil von 13,8 \% richtig beurteilter Beobachtungen innerhalb des ersten Laktationsmonats feststellen konnte. Seggewiß (2004) wertete die Laktationsmonate getrennt aus und schlussfolgerte, dass mit fortschreitender Laktationsdauer die richtigen Einschätzungen zunehmen: Mit Ende des dritten Monats konnten demnach 26,3\% korrekt beurteilt werden, im sechsten Laktationsmonat sogar 32,2 \%.

Spohr und Wiesner (1991) wiesen bei der Vorstellung der 9-Felder-Tafel darauf hin, dass bei dieser Form der Beurteilung die Energie- und Proteinversorgung in Relation zueinander und auch in Relation zur Milchleistung berücksichtigt werden muss. Die eingeführte Beurteilung auf Basis der Milchinhaltsstoffe lässt jedoch Letzteres unbeachtet (Losand et al., 2016). Der Milcheiweißgehalt sinkt mit steigender Milchleistung, weshalb fixe Grenzen als Indikator für die Energieversorgung ungünstig sind. Der FEQ wird demgegenüber von der produzierten Milchmenge nicht beeinflusst und ist deshalb als Indikator für die Versorgung mit Energie besser geeignet (Losand et al., 2016). Um die 9-Felder-Tafel auf ihre Validität hin zu bewerten, werteten Losand et al. (2016) die Daten der Milchleistungsprüfung in Mecklenburg-Vorpommern mithilfe linearer Regressionen aus, um einen Normalbereich für den Milcheiweißgehalt in Relation zur Milchleistung festzulegen. Dabei verwendeten sie nicht nur den Grenzwert des Eiweißgehaltes von 3,2\%, sondern bezogen sowohl die untere Grenze des berechneten Normalbereichs als auch den FEQ in ihre Auswertungen mit ein. Auf deren Basis erstellten sie eine neue grafische Darstellungsform mit nur noch sechs Feldern. Diese 6-Felder-Tafel soll eine Option 


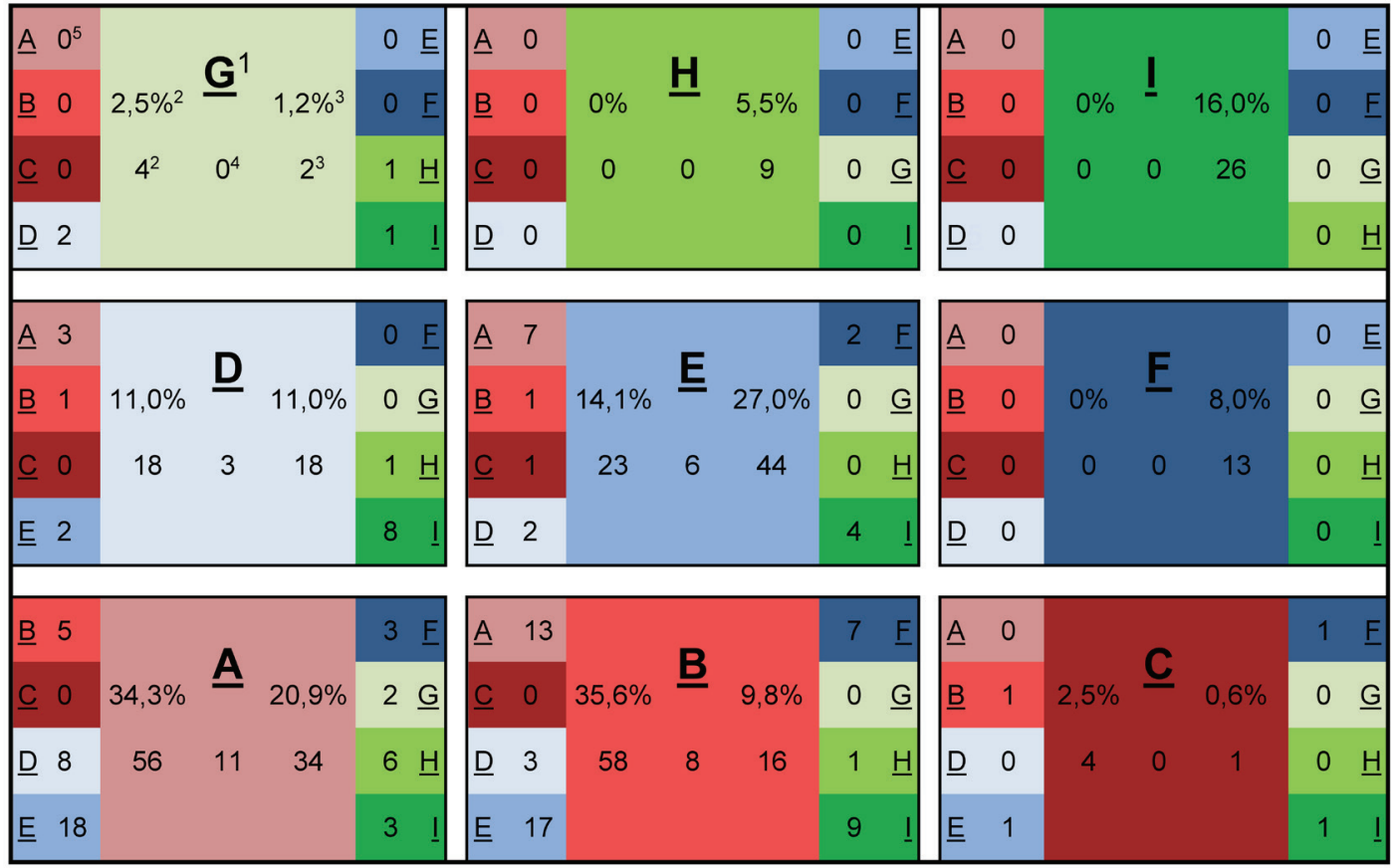

Abbildung 3. Zuordnung der Beobachtungen nach der 9-Felder-Tafel in den ersten zehn Laktationswochen

Figure 3. Allocation of observations according to the 9-field table during the first ten weeks of lactation

${ }^{1}$ Feld-Bezeichnung gemäß Schema in Abbildung 1; ${ }^{2}$ Anteil bzw. absolute Anzahl der Beobachtungen, die dem jeweiligen Feld nach Milchinhaltsstoffen zugeordnet werden; ${ }^{3}$ Anteil bzw. absolute Anzahl der Beobachtungen, die dem jeweiligen Feld nach der tatsächlichen Versorgungsbilanz zuzuordnen wären; ${ }^{4}$ Anzahl der übereinstimmenden Zuordnung zum jeweiligen Feld aufgrund von Milchinhaltsstoffen und tatsächlicher Versorgungsbilanz; ${ }^{5}$ absolute Anzahl der Beobachtungen, die gemäß Schema in das jeweilige Feld (1) eingeordnet wurden, nach der Versorgungslage aber zu jenem in der Randleiste bezeichneten Feld gehört

darstellen, um Versorgungslagen weit außerhalb des Optimums über die Milchinhaltstoffe erkennen zu können (Losand et al., 2016). Bei der Auswertung im Rahmen der vorliegenden Arbeit wurden durch die 6-Felder-Tafel $27,6 \%$ der Beobachtungen richtig eingeschätzt (Tabelle
5). Durch den Chi-Quadrat-Anpassungstest konnte für die 6-Felder-Tafel ebenfalls eine signifikante Abweichung $(\mathrm{P}<0,001)$ der Einschätzung von der erwarteten Verteilung hinsichtlich aller Felder festgestellt werden (Ergebnisse nicht dargestellt).

Tabelle 6. Sensitivität (Se), Spezifität (Sp), positive und negative Likelihood-Ratio (LR+, LR-) der Zuordnungen gemäß den Felder-Tafeln für die ersten zehn Laktationswochen (LW)

Table 6. Sensitivity (Se), Specificity (Sp), positive and negative likelihood ratio (LR +, LR-) of the allocations according to field tables for the first ten weeks of lactation (LW)

\begin{tabular}{|c|c|c|c|c|c|c|c|c|}
\hline \multirow[t]{2}{*}{ Gruppe } & \multicolumn{4}{|c|}{ 9-Felder-Tafel } & \multicolumn{4}{|c|}{ 6-Felder-Tafel } \\
\hline & $\mathrm{Se}$ & $S_{p}$ & LR+ & LR- & $\mathrm{Se}$ & $S p$ & LR+ & LR- \\
\hline A & 0,32 & 0,65 & 0,93 & 1,04 & 0,38 & 0,65 & 1,10 & 0,95 \\
\hline B & 0,50 & 0,66 & 1,47 & 0,76 & 0,56 & 0,79 & 2,67 & 0,55 \\
\hline C & 0,00 & 0,98 & 0,00 & 1,03 & 0,00 & 0,95 & 0,00 & 1,05 \\
\hline D & 0,17 & 0,90 & 1,61 & 0,93 & 0,40 & 0,92 & 4,77 & 0,65 \\
\hline $\mathbf{E}$ & 0,14 & 0,86 & 0,95 & 1,01 & 0,21 & 0,83 & 1,20 & 0,96 \\
\hline $\mathbf{F}$ & 0,00 & 1,00 & n. def. & 1,00 & 0,10 & 0,98 & 4,24 & 0,92 \\
\hline G & 0,00 & 0,98 & 0,00 & 1,03 & & & & \\
\hline $\mathbf{H}$ & 0,00 & 1,00 & n. def. & 1,00 & & & & \\
\hline I & 0,00 & 1,00 & n. def. & 1,00 & & & & \\
\hline
\end{tabular}


Die wesentlichen Unterschiede der 6- zur 9-Felder-Tafel sind, wie bereits erwähnt, die niedrigere Harnstoff-Obergrenze und die Heranziehung des FEQ zur Beurteilung der Energieversorgung anstatt des Eiweißgehaltes allein. Des Weiteren weist die 6-Felder-Tafel hinsichtlich der Beurteilung der Energieversorgung nur zwei verschiedene Klassen auf: eine Unter- und eine Optimalversorgung. Eine Überversorgung mit Energie wird bei der 6-FelderTafel aus zwei Gründen nicht angezeigt: Ein Milcheiweißgehalt über 3,8\% wird in der Regel von sehr niedrigen Milchleistungen über die gesamte Laktation begleitet. Solch niedrige Milchleistungen und folglich ein Anstieg der Inhaltsstoffe werden wahrscheinlich nicht durch die Überversorgung mit Energie verursacht, sondern durch Effekte wie Änderung der Fütterung, Brunst oder Erkrankungen. Eine Betrachtung des Einzeltieres ist in diesem Fall empfehlenswert. Zum anderen können hohe Milchleistungen nur bei hoher Energieversorgung erbracht werden, weshalb deren Beschränkung nicht zu empfehlen ist. Sinkt die Leistung allerdings im Laktationsverlauf, sind diese Tiere gefährdet, überversorgt zu werden (Losand et al., 2016). Im zugrunde liegenden Datenmaterial weisen nur vier Beobachtungen einen Eiweißgehalt über 3,8 \% auf, in zwei der drei Felder (H und I) der 9-Felder-Tafel, die eine Energieüberversorgung anzeigen, wurden keine Beobachtungen eingeordnet. Die Reduktion der Felder im Schema der 6-Felder-Tafel durch Zusammenfassung der an Energie über- und optimal versorgten Tiere scheint daher sinnvoll. Der geringe Informationsverlust durch weniger Felder kann durch verstärkte Managementmaßnahmen wie die Beurteilung der Körperkondition ausgeglichen werden (Losand et al., 2016).

Nach Lößner et al. (2018) haben jene Tiere, die nach der 6-Felder-Tafel als ausreichend mit Energie versorgt eingestuft werden, eine durchschnittliche negative Energiebilanz von -11,3 MJ NEL/Tag. Wird bei einem FEQ $<1,4$ die Energiebilanz von -32 MJ NEL nicht unterschritten, wird die Beobachtung als richtig positiv eingestuft. Dieser Anteil liegt bei $83,5 \%$. Bei den Beobachtungen, die aufgrund eines FEQ $\geq 1,4$ als energetisch unterversorgt eingestuft wurden, liegt der Anteil der korrekt eingeschätzten bei $65 \%$ (Lößner et al., 2018). In der vorliegenden Arbeit lag der Anteil korrekter Einschätzung bei separater Betrachtung der Energieversorgung bei 54 \% (Tabelle 3). Dabei sollte bedacht werden, dass die Grenze zwischen Optimal- und Unterversorgung in der vorliegenden Arbeit bei -10 \% des Tagesbedarfs an Energie gesetzt wurde. Bei jenen Beobachtungen, die eine Energiebilanz kleiner -32 MJ NEL haben, liegt die durchschnittliche Bedarfsdeckung bei $71 \%$, deutlich unter der angesetzten Grenze von -10\%. Diese Differenz dürfte den großen Unterschied hinsichtlich korrekter Beurteilung auf Basis des FEQ erklären. Hinsichtlich der Proteinversorgung stimmt die Einschätzung der 6-FelderTafel in der vorliegenden Arbeit zu 41,1 \% (Tabelle 5) mit der Versorgungsbilanz überein.

\subsection{Zuverlässigkeit der Einschätzung}

Die zur weiteren Auswertung verwendete grafische Darstellungsform ist in Abbildung 3 sowie Abbildung 4 ersichtlich. Die Felder der ursprünglichen Tafeln (Abbildungen 1 und 2) wurden mit den gleichen Buchstaben bezeichnet, wobei Feld A Protein- und Energiemangel darstellt. Eine Überversorgung mit Energie und Protein liegt bei der 9-FelderTafel für Zuordnungen zu Feld I vor, bei der 6-Felder-Tafel zu Feld F. Die Zahl unter der Feldbezeichnung gibt die absolute Anzahl der Beobachtungen an, bei denen die Beurteilung nach Milchinhaltsstoffen und die Versorgungsbilanz übereinstimmen. Daneben ist die Anzahl und über der Anzahl der Anteil der in dieses Feld eingeordneten Beobachtungen ersichtlich: auf der linken Seite auf Basis der Milchinhaltsstoffe und auf der rechten Seite nach der tatsächlichen Versorgungslage. Die unterstrichenen Buchstaben in den Randfeldern bezeichnen jene Beobachtungen, die gemäß Felder-Schema in das jeweilige Feld eingeordnet sind, aufgrund der Versorgungslage jedoch zu jenem in der Randleiste bezeichneten gehören.

In den Bereichen einer Überversorgung sowohl mit Energie als auch Protein befinden sich in der 9-Felder-Tafel sehr wenige Einschätzungen. Wie bereits erwähnt, weisen nur vier von 163 Beobachtungen einen Milcheiweißgehalt von über 3,8 \% auf. Nach der Versorgungsbilanz nehmen 37 Beobachtungen mehr als $110 \%$ ihres Energiebedarfs auf. Hinsichtlich Rohprotein gibt es ähnliche Werte (Abbildung 3): Wiederum vier Beobachtungen (Feld C) weisen einen Harnstoffgehalt größer 300 mg/l auf, 40 Beobachtungen stammen von mit Rohprotein überversorgten Kühen (Felder C, F, I). In den unteren Feldern (A, B, C) treten ebenfalls große Differenzen zwischen den eingeschätzten und den nach tatsächlicher Versorgungslage beurteilten Beobachtungen auf. Selbst wenn, wie in Feld $\mathrm{D}$, die Anzahl der Beobachtungen aufgrund beider Informationsquellen übereinstimmt, bedeutet dies nicht, dass dieses Feld eine besonders gute Einschätzungsgenauigkeit aufweist: Wie die mittige Zahl darstellt (Feld D) sind nur drei Beobachtungen korrekt beurteilt. 


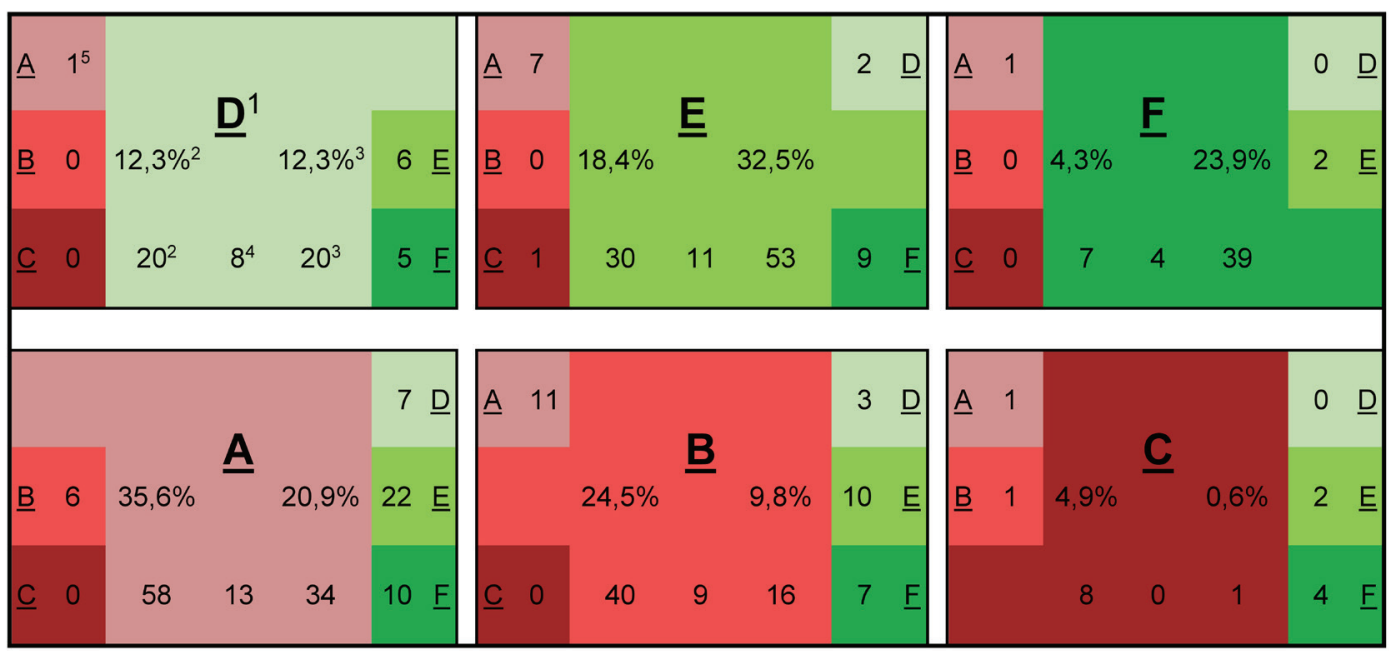

Abbildung 4. Zuordnung der Beobachtungen nach der 6-Felder-Tafel in den ersten zehn Laktationswochen Figure 4. Allocation of observations according to the 6-field table during the first ten weeks of lactation

${ }^{1}$ Feldbezeichnung gemäß Schema in Abbildung 2; ${ }^{2}$ Anteil bzw. absolute Anzahl der Beobachtungen, die dem jeweiligen Feld nach Milchinhaltsstoffen zugeordnet werden; ${ }^{3}$ Anteil bzw. absolute Anzahl der Beobachtungen, die dem jeweiligen Feld nach der tatsächlichen Versorgungsbilanz zuzuordnen wären; ${ }^{4}$ Anzahl der übereinstimmenden Zuordnung zum jeweiligen Feld aufgrund Milchinhaltsstoffen und tatsächlicher Versorgungsbilanz; ${ }^{5}$ Absolute Anzahl der Beobachtungen, die gemäß Schema in das jeweilige Feld (1) eingeordnet wurden, nach der Versorgungslage aber zu jenem in der Randleiste bezeichneten Feld gehört.

Die 6-Felder-Tafel (Abbildung 4) verteilt die Einschätzungen gleichmäßiger als die 9-Felder-Tafel, es gibt keine Felder ohne Zuordnungen. Durch die Herabsetzung der Harnstoff-Obergrenze von 300 auf $250 \mathrm{mg} / \mathrm{l}$ werden statt vier (9-Felder-Tafel) durch die 6-Felder-Tafel 15 Beobachtungen als proteinüberversorgt in den Feldern $\mathrm{C}$ und $\mathrm{F}$ eingeschätzt. Mit den 40 Beobachtungen, die tatsächlich überversorgt sind, stimmt dies nur viermal überein. Im Feld D fällt die Übereinstimmung von Einschätzung und tatsächlicher Versorgung auf, der Anteil der korrekt eingeschätzten Beobachtungen ist mit acht gering, wenn auch höher als bei der 9-Felder-Tafel. Die 9-Felder-Tafel bringt in den Feldern A, B E, F und I sehr große Differenzen zwischen der eingeschätzten und der nach Deckungsgrad zugeordneten Versorgungslage. Diese Unterschiede äußern sich auch in den sehr niedrigen Werten für Sensitivität und Likelihood-Ratios (Tabelle 6). Für fünf Felder der 9-Felder-Tafel sowie einem Feld der 6-Felder-Tafel beträgt die Sensitivität 0. Die positive Likelihood-Ratio ist bei der 9-Felder-Tafel in den ersten zehn Laktationswochen in keinem der Felder besser als „schlecht“. Bei der 6-Felder-Tafel zeichnet sich ein ähnliches Bild mit bestenfalls mäßigen Ergebnissen in den Feldern B, D und F ab. Die Spezifität ist für viele Felder akzeptabel, sie bewegt sich für beide Tafeln zwischen 0,65 und 1,00. Dies liegt vorwiegend daran, dass es aufgrund vieler unterschiedlicher Felder zahlreiche korrekt negative Beobachtungen gibt.

Aufgrund der wenig korrekten Einschätzung anhand der Felder-Tafeln sollte hinterfragt werden, wie gut die Milchinhaltsstoffe tatsächlich für die individuelle Beurteilung der Energie- und Proteinversorgungslage geeignet sind. Eicher et al. (1999) stellen fest, dass die Einordnung nach Spohr und Wiesner (1991) wesentliche Einflüsse, die das Einzeltier auf seinen Energie- und Proteinstoffwechsel hat, außer Acht lässt. Studien bestätigen allerdings, dass die Energieversorgung auf den Proteingehalt der Milch signifikant einwirkt (Kirchgeßner et al., 1986; Broderick, 2003) und der Milchharnstoffgehalt von der Proteinversorgung des Wiederkäuers beeinflusst wird (Recktenwald et al., 2014; Amanlou et al., 2017). Kirchgeßner et al. (1986) untersuchten die Auswirkungen der Protein- und Energieversorgung auf die Milchinhaltsstoffe und stellten fest, dass die Milchleistung unabhängig von der Proteinversorgung einen Einfluss auf die Harnstoff-Ausscheidung hat. Pro kg Anstieg der Milchleistung erhöhte sich der Milchharnstoffgehalt um $0,36 \%$. Für den Harnstoffwert wurde ein Optimalbereich zwischen 160 und $240 \mathrm{mg} / \mathrm{l}$ angegeben, wobei eine Änderung der Milchleistung um $5 \mathrm{~kg}$ - relativ zu $20 \mathrm{~kg}$ - den Milchharnstoffgehalt um $\pm 20 \mathrm{mg} / \mathrm{l}$ verändert. Wie bereits für den Milcheiweißgehalt beschrieben, 
sollen auch für den Milchharnstoffgehalt starre Grenzen (d. h. ohne Rücksicht auf die Milchleistung) keine treffende Aussage zur Versorgung erlauben. Der Milchharnstoffgehalt macht laut Losand et al. (2016) eine Aussage zur aktuellen Proteinversorgung des Tieres möglich. Nach Lößner et al. (2018) weist er keine plausible Relation zur Versorgungslage auf, wobei sehr niedrige Werte noch eher auf eine Unterversorgung hindeuten als höhere Gehalte allgemein brauchbare Informationen liefern. Hof et al. (1997) stellten in einer Untersuchung zur Eignung des Milchharnstoffgehaltes für die Einschätzung der Proteinversorgung fest, dass dieser eher zur Beurteilung des Protein/Energie-Verhältnisses taugt als zur Beurteilung der absoluten Proteinversorgungslage und dass die Beurteilung auf Herdenebene besser funktioniert als auf Einzeltierbasis. Dies deckt sich mit Ergebnissen von Lößner et al. (2018), die statt einer Aussage über die Proteinversorgung einen Zusammenhang zur täglichen ruminalen Stickstoffbilanz feststellen konnten, die über das nutzbare Rohprotein die zur Verfügung stehende Energie mit einbezieht. Ebenso konnten Schepers und Meijer (1998) aufgrund des Milchharnstoffgehaltes die Bilanz des unbeständigen Eiweißes gut einschätzen. Nach dem ersten Laktationsmonat konnte Seggewiß (2004) durch den Harnstoffgehalt akzeptable Ergebnisse zur Beurteilung der Versorgung mit Protein erzielen, im Gegensatz zu den oben erwähnten Quellen konnten jedoch keine statistisch gesicherten Zusammenhänge der Proteinbilanz und der ruminalen Stickstoffbilanz mit dem Milchharnstoffgehalt nachgewiesen werden. Darum konnte auf dessen Basis keine Einzeltierbeurteilung getroffen werden (Seggewiß, 2004).

Bei Heuer et al. (2001) und Friggens et al. (2007) konnte die Energiebilanz gut mithilfe der Milchinhaltsstoffe beurteilt werden. Allerdings wurde sie anhand von Gleichungen geschätzt, die sowohl Milchleistung, Proteingehalt, FEQ, Tage in Milch als auch weitere Faktoren mit einbezogen. Løvendahl et al. (2010) bezeichneten hingegen die Beurteilung der Energiebilanz aufgrund der Milchinhaltsstoffe als schwierig, da hohe Vorhersage-Fehler auftraten und die Wiederholbarkeit auch bei kurz aufeinanderfolgenden Bestimmungen der Milchinhaltsstoffe im Vergleich zur Beurteilung mittels Körperkondition und Lebendmasse schlecht erschienen. Die Energiebilanz konnte aufgrund der Milchinhaltsstoffe nur mäßig gut eingeschätzt werden und bot damit für die Einzeltierbeurteilung keine adäquate Methode.

In der Literatur bestehen also konträre Ansichten zur möglichen Nutzung der Milchinhaltsstoffe für die Beurteilung des Versorgungsstatus von Milchkühen. Um herauszufin- den, ob die in den Tafeln verwendeten Parameter nur mäßig geeignet sind oder ob eine Anpassung der Grenzwerte nötig ist, ist es wichtig zu wissen, welchen Feldern die falsch beurteilten Beobachtungen zugeordnet wurden.

\subsection{Betrachtung fehlerhafter Zuordnungen}

In den eine Überversorgung repräsentierenden Feldern $(\mathrm{C}$, F, G, $\mathrm{H}$ und I in Abbildung 3 bzw. $\mathrm{C}$ und F in Abbildung 4) sind keine bis wenige Einschätzungen nach den Milchinhaltsstoffen vorhanden, was auf zu hohe obere Grenzwerte hindeutet. Darum ist die Betrachtung, in welchen Feldern sich diese nach Versorgungsbilanz überversorgten Beobachtungen befinden, interessant. Stimmt die Theorie, dass die Grenzwerte - vor allem bezüglich des Harnstoffes (Seggewiß, 2004; Losand et al., 2016) - zu hoch sind, dann sollten sich die fehlenden Beobachtungen in den angrenzenden Feldern wiederfinden. Wird nun zuerst die 9-Felder-Tafel betrachtet, fällt auf, dass von jenen 45 Beobachtungen in Feld A, die falsch zugeordnet wurden, 31 in benachbarte Felder (B, D und E), 14 jedoch in die Felder $\mathrm{C}$ und $\mathrm{F}$ bis I gehören würden (Abbildung 3). Jene 26 Beobachtungen, die in Feld I eingeordnet sein sollten, teilen sich vorwiegend auf die Felder A, B, D und $\mathrm{E}$ auf, in den direkt angrenzenden Feldern $\mathrm{F}$ und $\mathrm{H}$ befinden sich dagegen keine derselben. Jene Beobachtungen, die laut Bilanz proteinüberversorgt sind, befinden sich in den Feldern A, E und vorwiegend B und D. Eine Absenkung des unteren Harnstoff-Grenzwertes würde sicherlich dazu führen, dass manche der 49 Beobachtungen, die hinsichtlich Harnstoffgehalt als unterversorgt eingeschätzt wurden, sich aber bezüglich Protein-Bilanz im Optimalund Überversorgungsbereich befinden, nun korrekt beurteilt würden. Allerdings blieben jene 25 Beobachtungen, die bereits jetzt als optimal versorgt beurteilt wurden (Abbildung 3), tatsächlich aber weniger als $90 \%$ ihres Tagesbedarfs an Rohprotein aufgenommen haben, falsch eingeschätzt. Wie viele derzeit korrekt Beurteilte durch einen niedrigeren Grenzwert zu einer falschen Einschätzung wechseln würden, wurde nicht festgestellt. Es sollte aber bedacht werden, dass falsche Beurteilungen nicht nur an einem zu hohen Grenzwert liegen, sondern auch Unterversorgte bereits jetzt nach den Milchinhaltsstoffgehalten als optimal versorgt eingestuft werden. So weisen $46,3 \%$ aller Proteinunterversorgten einen Milchharnstoffgehalt über $150 \mathrm{mg} / \mathrm{l}$ auf. Betreffend Energieversorgung liegt die Zahl jener, die als unterversorgt eingeschätzt wurden, tatsächlich aber optimal oder überversorgt sind, bei 80 Beobach- 
tungen (49,1\%), im Gegensatz zu nur 13 (7,9\%), welche trotz Aufnahme von weniger als $90 \%$ des Tagesbedarfs als optimal- oder überversorgt beurteilt wurden.

Ähnliches ist für die 6-Felder-Tafel festzustellen: Auch hier stammen jene 45 falsch in Feld A eingeordneten Beobachtungen aus den anderen Feldern B und D bis F (Abbildung 4). Umgekehrt gibt es auch 11 Beobachtungen in Feld B, die nach Versorgungslage Feld A zugeordnet sein sollten. Konkret befinden sich 30,1 \% der Beobachtungen trotz optimaler oder Überversorgung mit Protein im untersten Bereich (A, D) der Felder-Tafel. Bezüglich Energie wurden 39,9 \% trotz optimaler Aufnahme als mangelversorgt beurteilt.

Vor allem am Beginn der Laktation, in der die Erkennung von Fehlversorgungen besonders wichtig wäre, ist der Anteil der korrekten Beurteilungen der 9-Felder-Tafel sehr gering (Seggewiß, 2004), ebenso in der 6-Felder-Tafel. In den ersten zehn Laktationswochen geben beide Felder-Tafeln also eine dramatischere Einschätzung der Versorgungslage wieder, als tatsächlich vorliegt. Dabei kann argumentiert werden, dass eine strengere Einordung auch Vorteile hat, da Landwirte in der Praxis besser auch jene Tiere als unterversorgt einstufen, die - wenn überhaupt - erst an der Grenze zur Unterversorgung liegen. Da aber ein Großteil der Beobachtungen unzutreffend als energieunterversorgt und bzw. oder proteinunterversorgt eingeschätzt wird, fallen auch gut und sogar stark überversorgte Tiere unter diese fälschliche Zuordnung in kritisch mangelversorgte Felder. Dagegen werden auch Tiere mit tatsächlichem Mangel als optimal versorgt dargestellt. Ein Prognose-Tool, das fixe Grenzwerte verwendet, kann nicht alle Tiere korrekt beurteilen. Dies unterstreicht die Annahme, dass die Milchinhaltsstoffe weniger als Prognose-Instrument für die Beurteilung eines Einzeltieres geeignet sind als für das Anzeigen eines Herdentrends (Hof et al., 1997). Das wird durch die Aussagen von Kirchgeßner et al. (1986) und Losand et al. (2016) untermauert, dass manche Tiere durch einen genetisch determinierten (niedrigen) Proteingehalt durch ein Management-Tool wie die Felder-Tafel möglicherweise nicht korrekt beurteilt werden. Durch die Einbeziehung mehrerer Parameter (z. B. Laktationsdauer, Milchleistung, FEQ) in die Schätzung der Versorgungsbilanz (vor allem hinsichtlich Energie), konnte die Validität verbessert werden (Heuer et al., 2001; Friggens et al., 2007). Die Kombination von Fett- und Eiweißgehalt allein, wie es Losand et al. (2016) in der 6-Felder-Tafel umsetzten, scheint dabei nicht auszureichen. Beide Felder-Tafeln haben in der vorliegenden Arbeit einen zu geringen Anteil an korrekten Einschätzungen, um eine Verwendung auf Einzeltierbasis in der Praxis empfehlen zu können. Für weitere Untersuchungen wäre interessant, zu analysieren, wie Rationsänderungen die Milchinhaltsstoffe von Herden beeinflussen.

\section{Schlussfolgerungen}

Keine der beiden Tafeln ließ für die zugrundeliegenden Daten aus einem Exaktversuch eine befriedigende Einschätzung der individuellen Versorgungslage zu. Die 6-Felder-Tafel schneidet bei Kombination von Energieund Proteinversorgung um rund zehn Prozentpunkte besser ab als die 9-Felder-Tafel, was auf den um $50 \mathrm{mg} / \mathrm{l}$ niedrigeren Harnstoff-Grenzwert für die Einschätzung einer hohen Proteinversorgung und auf die Verwendung des Fett/Eiweiß-Quotienten anstelle des Eiweißgehalts als Indikator für die Energieversorgung zurückzuführen ist. Da sich einige der falsch eingeschätzten Beobachtungen nicht in den benachbarten Feldern befanden, ist eine pauschale Adaptierung der Grenzwerte nicht zielführend. Aufgrund der niedrigen Sensitivitäts-Werte der Zuordnungen und einer nach positiver Likelihood-Ratio bloß mäßiger oder schlechter Testaussage kann eine routinemäßige Anwendung der Felder-Tafeln auf Einzeltierbasis in der Praxis nicht empfohlen werden. Ob bei Versorgungslagen, die deutlich stärker vom Bedarf abweichen als die hier zugrunde liegenden, eine bessere Aussagekraft der Einschätzungen zu erwarten ist, kann anhand des vorliegenden Datensatzes nicht beantwortet werden. Dies sollte in weiteren, ähnlich konzipierten Untersuchungen erfolgen. Des Weiteren wäre kritisch zu untersuchen, ob die Felder-Tafeln für die Verwendung auf Herdenbasis adaptiert werden sollten.

\section{Literaturverzeichnis}

Amanlou, H., Farahani, T.A. und N.E. Farsuni (2017): Effects of rumen undegradable protein supplementation on productive performance and indicators of protein and energy metabolism in Holstein fresh cows. Journal of Dairy Science 100, 3628-3640.

Bender, R. (2001): Interpretation von Effizienzmaßen der Vierfeldertafel für Diagnostik und Behandlung. Medizinische Klinik 96, 116-121.

Eicher, R., Bouchard, E. und M. Bigras-Poulin (1999): Factors affecting milk urea nitrogen and protein concentrations in Quebec dairy cows. Preventive Veterinary Medicine 39, 53-63. 
Firk, R., Stamer, E., Junge, W. und J. Krieter (2002): Automation of oestrus detection in dairy cows: a review. Livestock Production Science 75, 219-232.

Friggens, N.C., Ridder, C. und P. Løvendahl (2007): On the use of milk composition measures to predict the energy balance of dairy cows. Journal of Dairy Science 90, 5453-5467.

Gerber, P.J., Uwizeye, A., Schulte, R.P.O., Opio, C.I. und I.J.M. de Boer (2014): Nutrient use efficiency: a valuable approach to benchmark the sustainability of nutrient use in global livestock production? Current Opinion in Environmental Sustainability 9-10, 122-130.

GfE (Gesellschaft für Ernährungsphysiologie - Ausschuss für Bedarfsnormen) (2001): Energie- und Nährstoffbedarf landwirtschaftlicher Nutztiere, Nr. 8: Empfehlungen zur Energie- und Nährstoffversorgung der Milchkühe und Aufzuchtrinder. DLG-Verlag, Frankfurt am Main, Deutschland, 135 S.

Gruber, L. (2019): Unveröffentlichte Versuchsergebnisse. HBLFA Raumberg-Gumpenstein, Irdning, Österreich.

Heuer, C., Van Straalen, W.M., Schukken, Y.H., Dirkzwager, A. und J.P.T.M. Noordhuizen (2001): Prediction of energy balance in high yielding dairy cows with test-day information. Journal of Dairy Science 84, 471-481.

Hof, G., Vervoorn, M.D. und P.J. Lenaers (1997): Milk urea nitrogen as a tool to monitor the protein nutrition of dairy cows. Journal of Dairy Science 80, 3333-3340.

Kirchgeßner, M. (1992): Tierernährung. 8. Aufl., DLGVerlags-GmbH, Frankfurt am Main, Deutschland.

Kirchgeßner, M., Kreuzer, M. und D.A. Roth-Maier (1986): Milk urea and protein content to diagnose energy and protein malnutrition of dairy cows. Archives of Animal Nutrition 36, 192-197.

Losand, B., Hartwig, S., Heinz, S. und A. Römer (2016): Nutzung der MLP-Ergebnisse zur Charakterisierung der Versorgungslage und des Tierwohls von Milchkühen - eine kritische Betrachtung. Tagungsband Forum Angewandte Forschung Fulda, 45-52.

Lößner, P., Engelhard, T., Riemann, E., Glatz, J. und B. Losand (2018): Milchinhaltsstoffe und Energiebilanz aus Futterverzehr und Leistung, sprechen sie mit einer
Zunge? Validierungsergebnisse aus Idener Fütterungsversuchen. Tagungsband Forum Angewandte Forschung Fulda, 45-49.

Løvendahl, P., Ridder, C. und N.C. Friggens (2010): Limits to prediction of energy balance from milk composition measures at individual cow level. Journal of Dairy Science 93, 1998-2006.

Makkar, H.P.S. (2016): Animal nutrition in a 360-degree view and a framework for future R\&D work: towards sustainable livestock production. Animal Production Science 56, 1561-1568.

Recktenwald, E.B., Ross, D.A., Fessenden, S.W., Wall, C.J. und M.E. Van Amburgh (2014): Urea-N recycling in lactating dairy cows fed diets with 2 different levels of dietary crude protein and starch with or without monensin. Journal of Dairy Science 97, 1611-1622.

Schepers, A.J. und R.G.M. Meijer (1998): Evaluation of the utilization of dietary nitrogen by dairy cows based on urea concentration in milk. Journal of Dairy Science 81, 579-584.

Schwarz, F.J. (2014): Rinderfütterung. In: Stangl, G.I., Schwarz, F.J., Roth, F.X., Südekum, K.-H. and K. Eder (Hrsg.): Kirchgessner Tierernährung. 14. Aufl., DLGVerlag, Frankfurt/Main, 357-510.

Seggewiß, S. (2004): Überprüfung der Bedeutung von Milchinhaltsstoffen für die Beurteilung der Energie-, Protein- und Strukturversorgung von HF-Kühen. Dissertation, Tierärztliche Hochschule Hannover.

Spohr, M. und H.-U. Wiesner (1991): Kontrolle der Herdengesundheit und Milchproduktion mit Hilfe der erweiterten Milchleistungsprüfung. Die Milchpraxis 29, 231-236.

Tamminga, S. (2003): Pollution due to nutrient losses and its control in European animal production. Livestock Production Science 84, 101-111.

Urdl, M., Gruber, L., Obritzhauser, W. und A. Schauer (2015): Metabolic parameters and their relationship to energy balance in multiparous Simmental, Brown Swiss and Holstein cows in the periparturient period as influenced by energy supply pre- and post-calving. Journal of Animal Physiology and Animal Nutrition 99, 174-189. 